\title{
A model of economic growth with physical and human capital: the role of time delays
}

\author{
Luca Gori* ${ }^{*}$ Luca Guerrini ${ }^{\dagger} \bullet$ Mauro Sodini ${ }^{\ddagger}$
}

August 8, 2016

\begin{abstract}
This article aims at analysing a two-sector economic growth model with discrete delays. The focus is on the dynamic properties of the emerging system. In particular, the work concentrates on the study of the stability properties of the stationary solution and the conditions under which oscillatory dynamics emerge (through Hopf bifurcations). In order to make the above results more readable, it was proposed the stability crossing curves technique to characterise the stability conditions in the plane of time delays. In addition, the article proposes some numerical simulations to illustrate the behaviour of the system when the stationary equilibrium is unstable.
\end{abstract}

Keywords growth models

Delay differential equations; Stability crossing curves; Two-sector economic

JEL Codes E32; O41

AMS Codes $\quad 34 \mathrm{~K} 18 ; 34 \mathrm{~K} 20$

The Solow growth model (Solow, 1956) with physical capital accumulation is a cornerstone in economic theory and several developments of it have been proposed in the course of time, especially to account for human capital accumulation (Mankiw et al., 1992) and environmental issues (the Green Solow model). A relevant assumption of both the one-sector and two-sector Solow models is that production and accumulation of physical and human capital take place immediately. However, this is one of the main reasons why explaining fluctuations within such a framework is prevented. By accounting for the stylised fact that these processes can actually require time, this article contributes to the literature by considering a two-sector Solow-type set up with a time-to-build technology including time delays between the initial investment and its production capacity. This assumption enables the Solow growth model to explain temporary or persistent fluctuations in economic variables.

${ }^{*}$ L. Gori, Department of Political Science, University of Genoa, Piazzale E. Brignole, 3a, I-16125 Genoa (GE), Italy, e-mail: luca.gori@unige.it or dr.luca.gori@gmail.com, tel.: +39010209 9503, fax: +390102095536.

${ }^{\dagger}$ L. Guerrini (corresponding author), Department of Management, Polytechnic University of Marche, Piazza Martelli 8, 60121, Ancona (AN), Italy, e-mail: luca.guerrini@univpm.it, tel.: +390712207055.

${ }^{\ddagger}$ M. Sodini, Department of Economics and Management, University of Pisa, Via Cosimo Ridolfi, 10, I-56124 Pisa (PI), Italy, e-mail: mauro.sodini@unipi.it, tel.: +390502216 234, fax: +390502210603. 


\section{Introduction}

Despite (or perhaps because of) its simplicity, the one-sector Solow growth model (Solow 1956) with physical capital accumulation continues to be at the hearth of both the theory and empirics of economic growth and development. Indeed, by using this framework scholars have been often able to capture noteworthy aspects of the convergence towards long-term stationary values of per capita income of countries. This is because of the assumption of declining marginal product of capital, which in turn leads to a reduction in gross investments and the rate of economic growth. With specific regard to the empirics of the Solow growth model, the leading article of Mankiw et al. (1992) "takes Robert Solow seriously" (Mankiw et al., 1992, p. 407) and analyses whether a version of the Solow growth model augmented with human capital (two-sector model) is able to fit cross-country data related to the international variation and convergence in the standard of living of countries. The conclusion of these authors is that the Solow model is an "excellent" set up to explain the reasons why some (poor) countries grow faster than other (rich) countries do, as its theoretical predictions are consistent with the empirical evidence.

From a theoretical point of view, there are interesting extensions produced over the years by several authors. We recall here the contributions of Mirman (1973) in discrete time and Merton (1975) in continuous time, introducing a stochastic component in the Solow model and characterising the asymptotic properties of the systems. These works were then reconsidered later, amongst others, by Prandini (1994) and Schenk-Hoppé and Schmalfuß (2001). Another interesting extension is represented by the introduction of a spatial component within the Solow model (the so-called spatial Solow growth model). From a mathematical point of view, the dynamics of the system in this case are described by a partial differential equation (Camacho and Zou, 2004; Capasso et al., 2010). A study of the formal properties of the Solow model extended to the case of dynamics described by a non-autonomous system and related to problems of population dynamics can also be found in Guerrini (2006). In addition, thanks to the burgeoning interest to environmental concerns, the Solow approach to economic growth has also become a useful tool to describe how the environment is related to the production process. This is the case of the so-called Green Solow model by Brock and Scott Taylor (2010), who show that one of the most influential empirical result in environmental economics, that is the Environmental Kuznets Curve, is consistent with the theoretical one-sector Solow growth model. Finally, we mention the recent work of Brianzoni et al. (2015) that represents one of the first attempts to explain oscillatory dynamics in a deterministic Solow-type set up.

However, in its original version, the Solow model is not able to capture (temporary or persistent) fluctuations in per capita income observed in the process of economic growth. In fact, the traditional growth theory of Solow attempts to explain the behaviour of trend by ignoring cyclical fluctuations, and variations around the trend are attributed to exogenous shocks. In order to get models able to explain short-term and long-term fluctuations, economic theory has developed models with nontrivial allocative problems (for instance, in an overlapping generations framework see the recent contributions of Chen and Li, 2013, who consider the effects of child policies on long-term outcomes, and Gori and Sodini, 2014, who study a model with endogenous labour supply and multiplicative external habits) or disequilibrium Keynesian-type models (Naimzada and Pireddu, 2014).

One of the limiting assumptions of the augmented Solow model (preventing to explain fluctuations) is that production and accumulation of human capital take place immediately. This may be viewed as a limitation as these processes can actually require time. The purpose of this article is to introduce a time-to-build technology in the classical two-sector Solow model (with physical and human capital accumulation) that includes time delays between the initial investment and its pro- 
duction capacity. The introduction of a time-to-build technology for human capital, in fact, seems to be a natural phenomenon in the process of accumulation and depreciation of it, given the time required for its formation and use in production. This hypothesis was introduced by Zak (1999) in a one-sector model with physical capital. However, in their works both Zak (1999) and Ferrara et al. (2013) conduct only a local analysis and establish the existence of a Hopf bifurcation. This kind of bifurcation explains the possibility of business cycles in these two frameworks but nothing is said about their stability (see, for example, Ferrara et al., 2014 for some formal results concerning the direction and stability of the Hopf bifurcation in a modified Solow model) and the behaviour of the dynamic system when the time delay goes beyond its bifurcation value.

More generally, the existence of a Hopf bifurcation determines the emergence of regular cycles (quasi-periodic behaviour) considered however by some scholars to be too simple to mimic business cycles behaviours observed in actual markets. Unfortunately, numerical simulations seem to reveal that the only relevant finding obtained in the unidimensional Solow model augmented with discrete delays is that by increasing the time delay there is an increase in the extent of fluctuations but the qualitative behaviours of trajectories do not change (after a quite long transient, in fact, a generic trajectory shows only one maximum value and one minimum value). This result confirms that the one-sector Solow model (even if augmented with time delays) cannot be used to explain temporary or persistent fluctuations in income. Results change dramatically, however, as the Solow growth model augmented with human capital accumulation and time delays becomes a useful set up able to describe temporary or persistent fluctuations in economic variables.

In recent years, some phenomena related to economic growth have been analysed by using methods of other disciplines. In particular, a promising field, which is known as physical economics, uses the typical tools of thermodynamics to explain both the state and possible fluctuations of the main macroeconomic variables. Based on the use of non-exact differential forms and the second law of thermodynamics, some scholars have developed models characterised by a few number of parameters but able to mimic some features of economic growth models, such as the existence of different growth rates of per capita GDP. In this regard, an interesting approach is the one related to the similarity that some authors establish between entropy and utility functions allowing some macroeconomic behaviours to be microfounded. (see Richmond et al., 2013 for a general reference). Another approach is the one proposed by Chakrabarti and Chatterjee (2004) and Chatterjee et al. (2003, 2004) with the aim of describing the behavior of trading on the markets. For doing this, the authors accounted for some fundamentals of the mathematical physics - specifically related to the interaction amongst molecules of the ideal-gas - to identify some models (the so-called kinetic models) showing results similar to those on income distribution.

The rest of the article proceeds as follows. Section 2 introduces a two-sector Solow growth model augmented with human capital accumulation and time delays. Section 3 provides an analysis of the stability properties of the dynamic system. Section 4 outlines the conclusions.

\section{The model}

The one-sector Solow growth model is a cornerstone of economic theory and it has been the object of several generalisations. Specifically, in order to validate it empirically, in an important contribution Mankiw et al. (1992) account for human capital accumulation. The dynamics of the model in that case are described by a two-dimensional continuous time dynamic system. However, some results of this augmented Solow model are based on the assumption that investments in physical capital and human capital and production of goods that can be used as productive inputs occur at the 
same time. Indeed, it is important to note that transforming investments in productive stocks of physical and human capital may require some gestation lags. As an example, one can think about education, infrastructures and innovation.

In order to account for these assumptions, we modify the model of Mankiw et al. (1992) by considering that the intensive production function at time $t$ has the following expression:

$$
y(t)=k\left(t-\tau_{1}\right)^{\alpha} h\left(t-\tau_{2}\right)^{\beta},
$$

where $k(t-\tau)$ and $h(t-\tau)$ are the stock of physical capital and the stock of human capital at time $t-\tau, y(t)$ is production at time $t, \alpha$ is the output elasticity of physical capital, $\beta$ is the output elasticity of human capital and $\alpha+\beta \in(0,1)$, implying that there exist decreasing returns in either forms of capital. ${ }^{1}$ Now, define $s_{k}$ and $s_{h}$ as the (exogenous) fractions of income invested in physical capital and human capital, respectively. By following the literature on time-to-build models, the time delay enters also (physical and human) capital depreciation (Zak, 1999). In addition, according with Mankiw et al. (1992) we let the depreciation rate $\delta_{k}=\delta_{h}=\delta$. By accounting for physical and human capital accumulation, the dynamics of the model are characterised by the following system of delay differential equations:

$$
\left\{\begin{array}{l}
\dot{k}=s_{k} k_{\tau_{1}}^{\alpha} h_{\tau_{2}}^{\beta}-\delta k_{\tau_{1}} \\
\dot{h}=s_{h} k_{\tau_{1}}^{\alpha} h_{\tau_{2}}^{\beta}-\delta h_{\tau_{2}}
\end{array},\right.
$$

where $k_{\tau_{1}}:=k\left(t-\tau_{1}\right)$ and $h_{\tau_{2}}:=h\left(t-\tau_{2}\right)$ (we omit the time index for simplicity). The equilibrium points of system (2) coincide with those of the corresponding system with no delays. Thus, there exists a unique steady state $\left(k_{*}, h_{*}\right)$, where

$$
k_{*}=\left(\frac{s_{k}^{1-\beta} s_{h}^{\beta}}{\delta}\right)^{\frac{1}{1-\alpha-\beta}} \text { and } h_{*}=\left(\frac{s_{k}^{\alpha} s_{h}^{1-\alpha}}{\delta}\right)^{\frac{1}{1-\alpha-\beta}} .
$$

In order to deal with the local stability of the equilibrium state we linearize system (2) around $\left(k_{*}, h_{*}\right)$ and get

$$
\left\{\begin{array}{l}
\dot{k}=(\alpha-1) \delta\left(k_{\tau_{1}}-k_{*}\right)+\left(\frac{\beta \delta s_{k}}{s_{h}}\right)\left(h_{\tau_{2}}-h_{*}\right), \\
\dot{h}=\left(\frac{\alpha \delta s_{h}}{s_{k}}\right)\left(k_{\tau_{1}}-k_{*}\right)+(\beta-1) \delta\left(h_{\tau_{2}}-h_{*}\right) .
\end{array}\right.
$$

The characteristic equation of system (3) can be expressed as follows

$$
\operatorname{det}\left(\begin{array}{cc}
(\alpha-1) \delta e^{-\lambda \tau_{1}}-\lambda & \frac{\beta \delta s_{k}}{s_{h}} e^{-\lambda \tau_{2}} \\
\frac{\alpha \delta s_{h}}{s_{k}} e^{-\lambda \tau_{1}} & (\beta-1) \delta e^{-\lambda \tau_{2}}-\lambda
\end{array}\right)=0,
$$

that is

$$
\lambda^{2}+b_{1} \lambda e^{-\lambda \tau_{1}}+c_{1} \lambda e^{-\lambda \tau_{2}}+d_{0} e^{-\lambda\left(\tau_{1}+\tau_{2}\right)}=0,
$$

where

$$
b_{1}=(1-\alpha) \delta>0, \quad c_{1}=(1-\beta) \delta>0, \quad d_{0}=(1-\alpha-\beta) \delta^{2}>0 .
$$

\footnotetext{
${ }^{1}$ We are assuming that the growth rates of exogenous technological progress and population are zero. We normalise the corresponding levels to one.
} 
In the coming sections, we shall analyze the distribution of the roots of Eq. (4) and the following Lemma is helpful in our investigation.

Lemma 1 For the transcendental equation

$$
\begin{aligned}
P\left(\lambda, e^{-\lambda \tau_{1}}, \ldots, e^{-\lambda \tau_{m}}\right)= & \lambda^{n}+p_{1}^{(0)} \lambda^{n-1}+\cdots+p_{n-1}^{(0)} \lambda+p_{n}^{(0)} \\
& +\left[p_{1}^{(1)} \lambda^{n-1}+\cdots+p_{n-1}^{(1)} \lambda+p_{n}^{(1)}\right] e^{-\lambda \tau_{1}}+\cdots \\
& \quad+\left[p_{1}^{(m)} \lambda^{n-1}+\cdots+p_{n-1}^{(m)} \lambda+p_{n}^{(m)}\right] e^{-\lambda \tau_{m}}=0,
\end{aligned}
$$

as $\left(\tau_{1}, \tau_{2}, \ldots, \tau_{m}\right)$ vary, the sum of orders of the zeros of $P\left(\lambda, e^{-\lambda \tau_{1}}, \ldots, e^{-\lambda \tau_{m}}\right)$ in the open right half plane can change only if a zero appears on or crosses the imaginary axis (see Ruan and Wei, 2003).

Preliminary, we observe that if $\tau_{1}=\tau_{2}=0$ then Eq. (4) becomes

$$
\lambda^{2}+\left(b_{1}+c_{1}\right) \lambda+d_{0}=0 .
$$

It is easy to see that Eq. (6) is solved by $\lambda=-(1-\alpha-\beta) \delta$ and $\lambda=-\delta$. All roots of (6) are negative. Hence, the equilibrium point $\left(k_{*}, h_{*}\right)$ is locally asymptotically stable.

\section{$3 \quad$ Stability analysis}

In this section we perform a stability analysis of dynamic system (2). In particular, Section 3.1 considers the case in which both time delays are positive and equal. Section 3.2 analyses the case in which one of the delay is zero and the other one varies. Section 3.3 takes into account the case of a positive time delay fixed in its stable interval by letting the other delay vary. Section 3.4 exemplifies the results obtained in the previous section by using the geometrical approach of the stability crossing curves. In addition, in order to clarify the behaviour of the dynamics after the equilibrium point is destabilised, this last section provides some numerical simulations.

\subsection{Case $\tau_{1}=\tau_{2}=\tau$}

The characteristic equation (4) takes the form

$$
P_{1}(\lambda, \tau):=\lambda^{2}+\left(b_{1}+c_{1}\right) \lambda e^{-\lambda \tau}+d_{0} e^{-2 \lambda \tau}=0 .
$$

In order to examine the existence of simple purely imaginary roots of (7) and the transversality at all corresponding bifurcation values, we use the following equation

$$
P(\lambda, \tau):=e^{\lambda \tau} P_{1}(\lambda, \tau)=e^{\lambda \tau} \lambda^{2}+\left(b_{1}+c_{1}\right) \lambda+d_{0} e^{-\lambda \tau}=0 .
$$

Substituting $\lambda=i \omega$, with $\omega>0$, into (8), we derive the real and imaginary parts

$$
\left\{\begin{array}{ccc}
\left(\omega^{2}-d_{0}\right) \cos \omega \tau & = & 0, \\
\left(\omega^{2}+d_{0}\right) \sin \omega \tau & = & \left(b_{1}+c_{1}\right) \omega .
\end{array}\right.
$$


Lemma 2 Eq. (8) has a pair of purely imaginary roots $\pm i \omega_{1}\left( \pm i \omega_{2}\right.$, respectively $)$ at $\tau=\tau_{1}^{j}\left(\tau=\tau_{2}^{j}\right.$, respectively), where

$$
\omega_{1,2}=\frac{b_{1}+c_{1} \pm \sqrt{\left(b_{1}+c_{1}\right)^{2}-4 d_{0}}}{2}, \quad \tau_{1,2}^{j}=\frac{1}{\omega_{1,2}}\left(\frac{\pi}{2}+2 \pi j\right),
$$

with $j=0,1,2, \ldots$

Proof. The statement follows from (9) considering the cases $\omega^{2}-d_{0}=0(\cos \omega \tau \neq 0)$ and $\omega^{2}-d_{0} \neq 0(\cos \omega \tau=0)$. Notice the former cannot occur since it gives $\sin \omega \tau=\left(b_{1}+c_{1}\right) \omega /\left(\omega^{2}+\right.$ $\left.d_{0}\right)=\left(b_{1}+c_{1}\right) /\left(2 \sqrt{d_{0}}\right)>1$. The latter implies $\omega^{2}-\left(b_{1}+c_{1}\right) \omega+d_{0}=0$, whose discriminant is $\left(b_{1}+c_{1}\right)^{2}-4 d_{0}=(\alpha+\beta)^{2} \delta^{2}>0$. Furthermore, it is $\omega_{1}<\omega_{2}$.

Let $\omega_{*} \in\left\{\omega_{1}, \omega_{2}\right\}$ be a root of system (9) and $\tau_{*}$ the corresponding value of $\tau$. We need to guarantee simple root and transversality at $\lambda=i \omega_{*}$ and $\tau=\tau_{*}$, respectively. Differentiating (8) with respect to $\tau$ yields

$$
\left[\lambda^{2} \tau e^{\lambda \tau}+2 \lambda e^{\lambda \tau}-d_{0} \tau e^{-\lambda \tau}+b_{1}+c_{1}\right]\left(\frac{d \lambda}{d \tau}\right)=\lambda\left(d_{0} e^{-\lambda \tau}-\lambda^{2} e^{\lambda \tau}\right) .
$$

Now, we use (11) to prove $\lambda=i \omega_{*}$ to be a simple root of (8). In fact, if it is not a simple root, then $\lambda\left(d_{0} e^{-\lambda \tau}-\lambda^{2} e^{\lambda \tau}\right)=0$. From (8) we obtain $2 \lambda e^{\lambda \tau}+b_{1}+c_{1}=0$. Hence,

$$
\cos \omega_{*} \tau_{*}=0 \text { and }-2 \omega_{*} \sin \omega_{*} \tau_{*}+b_{1}+c_{1}=0 .
$$

We will show that these identities yield a contradiction. If $\omega_{*}=\omega_{1}$, then $\cos \omega_{1} \tau_{1}=0$. From (9) and (12) we deduce $\omega_{1}^{2}-d_{0}=0$. The conclusion follows. Similarly, the case for $\omega_{*}=\omega_{2}$.

Next, from (11) we get

$$
\left(\frac{d \lambda}{d \tau}\right)^{-1}=\frac{2 \lambda e^{\lambda \tau}+b_{1}+c_{1}}{\lambda\left(d_{0} e^{-\lambda \tau}-\lambda^{2} e^{\lambda \tau}\right)}-\frac{\tau}{\lambda}
$$

which leads to

$$
\begin{aligned}
& \operatorname{sign}\left[\frac{d \operatorname{Re}(\lambda)}{d \tau}\right]_{\tau=\tau_{*}}=\operatorname{sign}\left[\operatorname{Re}\left(\frac{d \lambda}{d \tau}\right)^{-1}\right]_{\tau=\tau_{*}} \\
& =\operatorname{sign}\left\{\frac{2 \omega_{*}^{4}+2 \omega_{*}^{2} d_{0}\left(\cos ^{2} \omega_{*} \tau_{*}-\sin ^{2} \omega_{*} \tau_{*}\right)-\omega_{*}^{3}\left(b_{1}+c_{1}\right) \sin \omega_{*} \tau_{*}+\omega_{*} d_{0}\left(b_{1}+c_{1}\right) \sin \omega_{*} \tau_{*}}{\omega_{*}^{2}\left[\left(-\omega_{*}^{2}+d_{0}\right) \sin \omega_{*} \tau_{*}\right]^{2}+\omega_{*}^{2}\left[\left(\omega_{*}^{2}+d_{0}\right) \cos \omega_{*} \tau_{*}\right]^{2}}\right\} \\
& =\operatorname{sign}\{A\},
\end{aligned}
$$

where

$$
A=2 \omega_{*}^{3}+2 \omega_{*} d_{0}\left(\cos ^{2} \omega_{*} \tau_{*}-\sin ^{2} \omega_{*} \tau_{*}\right)-\omega_{*}^{2}\left(b_{1}+c_{1}\right) \sin \omega_{*} \tau_{*}+d_{0}\left(b_{1}+c_{1}\right) \sin \omega_{*} \tau_{*} .
$$

Recalling that $\cos \omega_{*} \tau_{*}=0$ and $\sin \omega_{*} \tau_{*}=1$, one has

$$
\operatorname{sign}\{A\}=\operatorname{sign}\left\{\left(b_{1}+c_{1}\right) \omega_{*}^{2}-4 d_{0} \omega_{*}+d_{0}\left(b_{1}+c_{1}\right)\right\}>0 .
$$


Notice that $\left(b_{1}+c_{1}\right) \omega_{*}^{2}-4 d_{0} \omega_{*}+d_{0}\left(b_{1}+c_{1}\right)=0$ has discriminant equal to $-4 d_{0}\left[\left(b_{1}+c_{1}\right)^{2}-4 d_{0}\right]<$ 0 . Hence $\operatorname{sign}\{A\}$ is positive. In conclusion

$$
\operatorname{sign}\left[\frac{d \operatorname{Re}(\lambda)}{d \tau}\right]_{\tau=\tau_{*}}>0 .
$$

From the previous discussions and the Hopf bifurcation theorem, we can obtain the following result.

Theorem 3 Let $\tau_{2}^{0}$ be defined as in (10). The equilibrium $\left(k_{*}, h_{*}\right)$ of system $(2)$ is locally asymptotically stable for $\tau \in\left[0, \tau_{2}^{0}\right)$ and unstable for $\tau>\tau_{2}^{0}$. System (2) undergoes a Hopf bifurcation at the equilibrium $\left(k_{*}, h_{*}\right)$ for $\tau=\tau_{2}^{0}$.

\subsection{Case $\tau_{1}=0, \tau_{2}>0$}

Eq. (4) becomes

$$
\lambda^{2}+b_{1} \lambda+\left(c_{1} \lambda+d_{0}\right) e^{-\lambda \tau_{2}}=0
$$

For $\omega>0$, we assume $\lambda=i \omega$ is a root of (13) to detect the critical value of stability transition. Then we get

$$
\omega^{2}=c_{1} \omega \sin \omega \tau_{2}+d_{0} \cos \omega \tau_{2}, \quad b_{1} \omega=d_{0} \sin \omega \tau_{2}-c_{1} \omega \cos \omega \tau_{2},
$$

which leads to

$$
\omega^{4}+\left(b_{1}^{2}-c_{1}^{2}\right) \omega^{2}-d_{0}^{2}=0
$$

where

$$
b_{1}^{2}-c_{1}^{2}=(\beta-\alpha)(2-\alpha-\beta) \delta^{2}, \quad d_{0}^{2}=(1-\alpha-\beta)^{2} \delta^{4}>0 .
$$

Let $z=\omega^{2}$, then (15) becomes

$$
z^{2}+\left(b_{1}^{2}-c_{1}^{2}\right) z-d_{0}^{2}=0 .
$$

We have the following conclusions.

Lemma 4 Eq. (15) has a unique positive root $\omega_{+}$, where

$$
\omega_{+}=\sqrt{\frac{-\left(b_{1}^{2}-c_{1}^{2}\right)+\sqrt{\left(b_{1}^{2}-c_{1}^{2}\right)^{2}+4 d_{0}^{2}}}{2}}
$$

and we can get the sequence of critical values of $\tau_{2}$ :

$$
\tau_{2, j}=\frac{1}{\omega_{+}}\left\{\cos ^{-1}\left[\frac{\left(d_{0}-b_{1} c_{1}\right) \omega_{+}^{2}}{c_{1}^{2} \omega_{+}^{2}+d_{0}^{2}}\right]+2 j \pi\right\}, \quad j=0,1,2, \ldots
$$

Proof. The first part of the statement comes from solving Eq. (16). From (14), we derive

$$
\sin \left(\omega_{+} \tau_{2}\right)=\frac{\left(b_{1} d_{0}+c_{1}^{2} \omega_{+}\right) \omega_{+}}{c_{1}^{2} \omega_{+}^{2}+d_{0}^{2}}>0, \quad \cos \left(\omega_{+} \tau_{2}\right)=\frac{\left(d_{0}-b_{1} c_{1}\right) \omega_{+}^{2}}{c_{1}^{2} \omega_{+}^{2}+d_{0}^{2}} .
$$

The critical values of $\tau$ for which the characteristic equation (13) has purely imaginary roots can now be determined from (18). 
Let $\lambda\left(\tau_{2}\right)$ be the root of (13) satisfying $\operatorname{Re}\left(\lambda\left(\tau_{2}\right)\right)=0$ and $\operatorname{Im}\left(\lambda\left(\tau_{2}\right)\right)=\omega_{+}$. Substituting $\lambda\left(\tau_{2}\right)$ into the left hand side of (13) and taking derivative with respect to $\tau_{2}$, we have

$$
\left[2 \lambda+b_{1}+c_{1} e^{-\lambda \tau_{2}}-\left(c_{1} \lambda+d_{0}\right) \tau_{2} e^{-\lambda \tau_{2}}\right]\left(\frac{d \lambda}{d \tau_{2}}\right)=\left(c_{1} \lambda+d_{0}\right) \lambda e^{-\lambda \tau_{2}}
$$

which yields

$$
\left(\frac{d \lambda}{d \tau_{2}}\right)^{-1}=\frac{\left(2 \lambda+b_{1}\right) e^{\lambda \tau_{2}}+c_{1}}{\left(c_{1} \lambda+d_{0}\right) \lambda}-\frac{\tau_{2}}{\lambda}
$$

Using (13) we have

$$
\operatorname{sign}\left\{\left.\frac{d(\operatorname{Re} \lambda)}{d \tau_{2}}\right|_{\substack{\tau_{2=\tau_{2, j}}=\omega_{+} \\ \omega=\omega_{+}}}\right\}=\operatorname{sign}\left\{\operatorname{Re}\left(\frac{d \lambda}{d \tau_{2}}\right)_{\substack{\tau_{2}=\tau_{2, j} \\ \omega=\omega_{+}}}^{-1}\right\}=\operatorname{sign}\left\{\frac{c_{1}^{2} \omega_{+}^{4}+2 d_{0}^{2} \omega_{+}^{2}+b_{1}^{2} d_{0}^{2}}{\left(c_{1}^{2} \omega_{+}^{2}+d_{0}^{2}\right)\left(\omega_{+}^{2}+b_{1}^{2}\right) \omega_{+}^{2}}\right\}>0 .
$$

The root $\lambda\left(\tau_{2}\right)$ of (13) crosses the imaginary axis from left to right at $\tau_{2}=\tau_{2, j}$ as $\tau_{2}$ increases. Furthermore, observe that $\lambda=i \omega_{+}$is a simple root of Eq. (13). If it is not simple, then from (19) we must have $\left(c_{1} i \omega_{+}+d_{0}\right) i \omega_{+} e^{-i \omega_{+} \tau_{2, j}}=0$, which is a contradiction. In view of above analysis, we arrive at the following conclusions.

Theorem 5 Let $\tau_{2, j}(j=0,1,2, \ldots)$ be defined as in (17). The equilibrium $\left(k_{*}, h_{*}\right)$ of system (2) is locally asymptotically stable when $\tau_{2} \in\left[0, \tau_{2,0}\right)$ and unstable when $\tau>\tau_{2,0}$. Moreover, system (2) undergoes Hopf bifurcations at the equilibrium for $\tau_{2}=\tau_{2, j}(j=0,1,2, \ldots)$.

\subsection{Case $\tau_{1}>0, \tau_{2} \in\left[0, \tau_{2,0}\right)$}

In order to study this case, we consider Eq. (4) with $\tau_{2}$ lying in its stable interval, i.e. $\tau_{2}=\tau_{2}^{*} \in$ $\left[0, \tau_{2,0}\right)$, and regarding $\tau_{1}$ as a parameter. Let $\lambda=i \omega(\omega>0)$ be a root of (4), then we have

$-\omega^{2}+b_{1} i \omega\left(\cos \omega \tau_{1}-i \sin \omega \tau_{1}\right)+c_{1} i \omega\left(\cos \omega \tau_{2}^{*}-i \sin \omega \tau_{2}^{*}\right)+d_{0}\left[\cos \omega\left(\tau_{1}+\tau_{2}^{*}\right)-i \sin \omega\left(\tau_{1}+\tau_{2}^{*}\right)\right]=0$.

Rewriting this equation according to its real and imaginary parts, we have

$$
\begin{aligned}
-\omega^{2}+b_{1} \omega \sin \omega \tau_{1}+c_{1} \omega \sin \omega \tau_{2}^{*} & =-d_{0} \cos \omega\left(\tau_{1}+\tau_{2}^{*}\right), \\
b_{1} \omega \cos \omega \tau_{1}+c_{1} \omega \cos \omega \tau_{2}^{*} & =d_{0} \sin \omega\left(\tau_{1}+\tau_{2}^{*}\right) .
\end{aligned}
$$

Using the square condition and adding the equations, we obtain

$$
\left(-\omega^{2}+b_{1} \omega \sin \omega \tau_{1}+c_{1} \omega \sin \omega \tau_{2}^{*}\right)^{2}+\left(b_{1} \omega \cos \omega \tau_{1}+c_{1} \omega \cos \omega \tau_{2}^{*}\right)^{2}=d_{0}^{2},
$$

namely

$$
g(\omega)=\omega^{4}+\left(-2 b_{1} \sin \omega \tau_{1}-2 c_{1} \sin \omega \tau_{2}^{*}\right) \omega^{3}+\left[b_{1}^{2}+c_{1}^{2}+2 b_{1} c_{1} \cos \omega\left(\tau_{1}-\tau_{2}^{*}\right)\right] \omega^{2}-d_{0}^{2}=0 .
$$

Notice that $g(0)=-d_{0}^{2}<0$ and $g(\omega)=+\infty$ as $\omega \rightarrow+\infty$. Therefore, Eq. (22) has at least a positive solution. Henceforth, assume Eq. (22) has finitely many positive roots $\omega_{1}, \omega_{2}, \ldots, \omega_{N}$. For every fixed $\omega_{l}, l=1,2, \ldots, N$, there exists a sequence $\tau_{1, l}^{j}>0(j=0,1,2, \ldots)$. Let

$$
\tilde{\tau}_{1}=\min \left\{\tau_{1, l}^{j}, l=1,2, \ldots, N, j=0,1,2, \ldots\right\} .
$$


For $\tau_{1}=\tilde{\tau}_{1}$, Eq. (4) has a pair of purely imaginary roots $\pm i \tilde{\omega}$. Let $\lambda\left(\tau_{1}\right)$ be the root of Eq. (4) near $\tau_{1}=\tilde{\tau}_{1}$ satisfying $\operatorname{Re}\left(\lambda\left(\tilde{\tau}_{1}\right)\right)=0, \operatorname{Im}\left(\lambda\left(\tilde{\tau}_{1}\right)\right)=\tilde{\omega}$. Then, by differentiating the two sides of Eq. (4) with respect to $\tau_{1}$, we obtain

$$
\begin{gathered}
{\left[2 \lambda+b_{1} e^{-\lambda \tau_{1}}+c_{1} e^{-\lambda \tau_{2}^{*}}-c_{1} \lambda \tau_{2}^{*} e^{-\lambda \tau_{2}^{*}}-d_{0}\left(\tau_{1}+\tau_{2}^{*}\right) e^{-\lambda\left(\tau_{1}+\tau_{2}^{*}\right)}-b_{1} \tau_{1} \lambda e^{-\lambda \tau_{1}}\right]\left(\frac{d \lambda}{d \tau_{1}}\right)} \\
=\lambda\left[b_{1} \lambda e^{-\lambda \tau_{1}}+d_{0} e^{-\lambda\left(\tau_{1}+\tau_{2}^{*}\right)}\right] .
\end{gathered}
$$

Thus,

$$
\left(\frac{d \lambda}{d \tau_{1}}\right)^{-1}=\frac{2 \lambda+b_{1} e^{-\lambda \tau_{1}}+c_{1} e^{-\lambda \tau_{2}^{*}}-c_{1} \lambda \tau_{2}^{*} e^{-\lambda \tau_{2}^{*}}-d_{0} \tau_{2}^{*} e^{-\lambda\left(\tau_{1}+\tau_{2}^{*}\right)}}{\lambda\left[b_{1} \lambda e^{-\lambda \tau_{1}}+d_{0} e^{-\lambda\left(\tau_{1}+\tau_{2}^{*}\right)}\right]}-\frac{\tau_{1}}{\lambda} .
$$

According to (4), one has that (25) rewrites as

$$
\left(\frac{d \lambda}{d \tau_{1}}\right)^{-1}=-\frac{2 \lambda+b_{1} e^{-\lambda \tau_{1}}+c_{1} e^{-\lambda \tau_{2}^{*}}+\tau_{2}^{*}\left(\lambda^{2}+b_{1} \lambda e^{-\lambda \tau_{1}}\right)}{\lambda\left(\lambda^{2}+c_{1} \lambda e^{-\lambda \tau_{2}^{*}}\right)}-\frac{\tau_{1}}{\lambda} .
$$

Then,

$$
\left(\frac{d \lambda}{d \tau_{1}}\right)_{\tau_{1}=\tilde{\tau}_{1}}^{-1}=\frac{A_{1}+i A_{2}}{\tilde{\omega}\left(B_{1}-i B_{2}\right)}-\frac{\tilde{\tau}_{1}}{i \tilde{\omega}}
$$

with

$$
\begin{aligned}
& A_{1}=b_{1} \cos \tilde{\omega} \tilde{\tau}_{1}+c_{1} \cos \tilde{\omega} \tau_{2}^{*}+\tau_{2}^{*}\left(b_{1} \tilde{\omega} \sin \tilde{\omega} \tilde{\tau}_{1}-\tilde{\omega}^{2}\right), \quad B_{1}=c_{1} \tilde{\omega} \cos \tilde{\omega} \tau_{2}^{*}, \\
& A_{2}=2 \tilde{\omega}-b_{1} \sin \tilde{\omega} \tilde{\tau}_{1}-c_{1} \sin \tilde{\omega} \tau_{2}^{*}+b_{1} \tilde{\omega} \tau_{2}^{*} \cos \tilde{\omega} \tilde{\tau}_{1}, \quad B_{2}=c_{1} \tilde{\omega} \sin \tilde{\omega} \tau_{2}^{*}-\tilde{\omega}^{2} .
\end{aligned}
$$

Therefore, we have

$$
\operatorname{sign}\left[\frac{d \operatorname{Re}(\lambda)}{d \tau_{1}}\right]_{\tau_{1}=\tilde{\tau}_{1}}=\operatorname{sign}\left[\operatorname{Re}\left(\frac{d \lambda}{d \tau_{1}}\right)^{-1}\right]_{\tau_{1}=\tilde{\tau}_{1}}=\operatorname{sign}\left(G\left(\tilde{\omega}, \tilde{\tau}_{1}\right)\right),
$$

where

$$
\begin{aligned}
G\left(\tilde{\omega}, \tilde{\tau}_{1}\right)=A_{1} B_{1}-A_{2} B_{2}=\left(2 \tilde{\omega}^{2}\right. & \left.+c_{1}^{2}\right) \tilde{\omega}-b_{1}^{2} \tilde{\omega} \sin \tilde{\omega} \tilde{\tau}_{1}-3 c_{1} \tilde{\omega}^{2} \sin \tilde{\omega} \tau_{2}^{*}+b_{1} c_{1} \tilde{\omega} \cos \tilde{\omega}\left(\tilde{\tau}_{1}-\tau_{2}^{*}\right) \\
& +\tau_{2}^{*}\left\{b_{1} \tilde{\omega}^{3} \cos \tilde{\omega} \tilde{\tau}_{1}-c_{1} \tilde{\omega}^{3} \cos \tilde{\omega} \tau_{2}^{*}+b_{1} c_{1} \sin \tilde{\omega}\left(\tilde{\tau}_{1}-\tau_{2}^{*}\right)\right\} .
\end{aligned}
$$

If $A_{1} B_{1}-A_{2} B_{2}>0$ (resp. $A_{1} B_{1}-A_{2} B_{2}<0$ ), then the pair of purely imaginary roots crosses the imaginary axis from left (resp. right) to right (resp. left) at $\tilde{\tau}_{1}$. It remains to prove $\lambda=i \tilde{\omega}$ to be a simple root of (4). Suppose this root is a repeated root. Then, (24) implies $b_{1} i \tilde{\omega} e^{-i \tilde{\omega} \tau_{1}}+$ $d_{0} e^{-i \tilde{\omega}\left(\tau_{1}+\tau_{2}^{*}\right)}=0$. Using (4), this means $i \tilde{\omega}\left(i \tilde{\omega}+c_{1} e^{-i \tilde{\omega} \tau_{2}^{*}}\right)=0$, and so $i\left(\tilde{\omega}-c_{1} \sin \tilde{\omega} \tau_{2}^{*}\right)+$ $c_{1} \cos \tilde{\omega} \tau_{2}^{*}=0$. Consequently, we must have $\tilde{\omega}=c_{1} \sin \tilde{\omega} \tau_{2}^{*}$ and $\cos \tilde{\omega} \tau_{2}^{*}=0$, i.e. $\tilde{\omega}=c_{1}$, $\sin \tilde{\omega} \tau_{2}^{*}=1$ and $\cos \tilde{\omega} \tau_{2}^{*}=0$. Recalling that $\cos \omega\left(\tau_{1}+\tau_{2}^{*}\right)=\cos \omega \tau_{1} \cos \omega \tau_{2}^{*}-\sin \omega \tau_{1} \sin \omega \tau_{2}^{*}$ and $\sin \omega\left(\tau_{1}+\tau_{2}^{*}\right)=\sin \omega \tau_{1} \cos \omega \tau_{2}^{*}+\cos \omega \tau_{1} \sin \omega \tau_{2}^{*}$, we derive from (20),(21) that $\sin \omega \tau_{1}=0$ and $\cos \omega \tau_{1}=0$, which is clearly a contradiction.

According to the general Hopf bifurcation theorem for FDEs in Hale (1977), we derive the following results on the stability and Hopf bifurcation of system (2). 
Theorem 6 Let $\tilde{\tau}_{1}, A_{1}, B_{1}, A_{2}, B_{2}$ and $G\left(\tilde{\omega}, \tilde{\tau}_{1}\right)$ be defined as in (23), (26), (27) and (28), $\tau_{2} \in$ $\left[0, \tau_{2,0}\right)$.

1) If Eq. (22) has no positive root, then the equilibrium $\left(k_{*}, h_{*}\right)$ of system (2) is locally asymptotically stable for $\tau_{1} \geq 0$.

2) If Eq. (22) has one positive root $\tilde{\omega}$, then

a) if $G\left(\tilde{\omega}, \tilde{\tau}_{1}\right)>0$, the equilibrium $\left(k_{*}, h_{*}\right)$ of system (2) is locally asymptotically stable for $\tau_{1} \in\left[0, \tilde{\tau}_{1}\right)$ and the equilibrium point loses its stability via a Hopf bifurcation at $\tau_{1}=\tilde{\tau}_{1}$;

b) if $G\left(\tilde{\omega}, \tilde{\tau}_{1}\right)<0$, the equilibrium $\left(k_{*}, h_{*}\right)$ of system (2) is locally asymptotically stable for $\tau_{1} \geq 0$.

3) If Eq. (22) has at least two positive roots, then there may exist a lot of stability switches and the stability of $\left(k_{*}, h_{*}\right)$ can change a finite number of times, at most, and eventually it becomes unstable.

a) If $G\left(\tilde{\omega}, \tilde{\tau}_{1}\right)>0$ the equilibrium $\left(k_{*}, h_{*}\right)$ of system (2) is locally asymptotically stable for $\tau_{1} \in\left[0, \tilde{\tau}_{1}\right)$ and system (2) undergoes a Hopf bifurcation at the equilibrium $\left(k_{*}, h_{*}\right)$ for $\tau_{1}=\tilde{\tau}_{1}$. The equilibrium $\left(k_{*}, h_{*}\right)$ remains unstable for $\tau_{1}>\tilde{\tau}_{1}$ until $\tau_{1}$ crosses a value $\tau_{1}=\tau_{1, l}^{j}$ such that $G\left(\omega_{l}, \tau_{1, l}^{j}\right)<0$. If $\hat{\tau}_{1}$ is this stability switch, then a Hopf bifurcation occurs at $\tau_{1}=\hat{\tau}_{1}$. As $\tau_{1}$ increases, the dynamic system may undergo a finite number of stability switches.

b) If $G\left(\tilde{\omega}, \tilde{\tau}_{1}\right)<0$, the equilibrium $\left(k_{*}, h_{*}\right)$ of system (2) remains locally asymptotically stable when $\tau_{1}$ crosses $\tilde{\tau}_{1}$ and it becomes unstable when $\tau_{1}$ crosses a value $\tau_{1}=\tau_{1, l}^{j}$ such that $G\left(\omega_{l}, \tau_{1, l}^{j}\right)>0$. A Hopf bifurcation occurs at this value. As $\tau_{1}$ increases, the dynamic system may undergo a finite number of stability switches.

\subsection{Stability crossing curves}

The conditions expressed in Theorem 6 are difficult to be read as $\operatorname{sign}\left(A_{1} B_{1}-A_{2} B_{2}\right)$ depends not only on the parameters of the model but also on the value of the time delay $\tau_{2}^{*}$ fixed in the interval $\left[0, \tau_{2,0}\right)$ as well as on $\tilde{\tau}_{1}$ defined in (23) and the corresponding $\tilde{\omega}$ obtained by (4). In order to give a clearer understanding of the dynamic properties of the model, a useful tool is represented by the stability crossing curves, introduced by Gu et al. (2005) and extended by Lin and Wang (2012) to study models with discrete delays in which in the characteristic equation also appears the term $e^{-\lambda\left(\tau_{1}+\tau_{2}\right)}$, as in (4). The first step in this procedure is to define the intervals for $\omega$ such that complex conjugate roots exist. Specifically, this coincides with the set of $\omega$ such that the following function, defined by starting from the characteristic equation (4),

$$
G(\omega):=\left(\delta^{2}-\omega^{2}\right)\left[\delta^{2}(1-\alpha-\beta)^{2}-\omega^{2}\right]\left\{\left[\delta^{2}(\alpha+\beta-1)+\omega^{2}\right]^{2}-(\alpha-\beta)^{2} \delta^{2} \omega^{2}\right\}
$$

is negative. We note that due to the simplicity of Eq. (4), $G(\omega)$ has an elementary expression allowing us to get such intervals in explicit form. 
Proposition $7 G(\omega)<0$ if and only if $\omega \in T$, where

$$
T:=(a \delta, b \delta) \cup(\delta(1-\alpha-\beta), \delta),
$$

where

$$
a=\frac{(\beta-\alpha) \operatorname{sign}(\beta-\alpha)+\sqrt{(\alpha-\beta)^{2}+4(1-\alpha-\beta)}}{2},
$$

and

$$
b=\frac{(\alpha-\beta) \operatorname{sign}(\beta-\alpha)+\sqrt{(\alpha-\beta)^{2}+4(1-\alpha-\beta)}}{2} .
$$

Therefore, it is possible to identify a parametric representation (with respect to $\omega$ ) in $\left(\tau_{1}, \tau_{2}\right.$ )plane of curves that mark the start of the birth or death of conjugate complex roots. Figures 1(a) and 1(b) illustrate the case when $s_{h}=0.24, s_{k}=0.2, \alpha=0.4, \beta=0.35$ and $\delta=0.35$. The stability crossing curves define a bounded set in $\left(\tau_{1}, \tau_{2}\right)$-plane in which the system is stable (the yellow region). A typical trajectory convergent towards the stationary equilibrium is depicted in Figure 2(a) for $\tau_{2}=9.28$. Too high values of any of the two delays cause the destabilisation of the equilibrium point (see Figure 2(b) that illustrates a limit cycle for $\tau_{2}=12$ ). These results characterise the local properties of the model around $\left(k_{*}, h_{*}\right)$. It is now interesting to note that, according to the parameter setting, it is possible to have different dynamic phenomena by moving away from the bifurcation values of the delays. In particular, there are cases in which the invariant curve increases its length until a threshold value $\tau_{2}$ beyond which the invariant curve is destroyed or it loses its stability. After this event, feasible trajectories do not exist for the system. Also, there may exist other parameter settings such that the projection of the invariant curve on the pseudo phase plane $(k(t), h(t))$ becomes self-intersecting when $\tau_{2}$ increases (Figure 2(c)). After a sufficiently long transient, trajectories of both $k(t)$ and $h(t)$ are characterised by the existence of two (relative) maximum values and minimum values. Increasing the value of $\tau_{2}$ further implies that these intersections increase (as well as the number of relative maximum values and minimum values, as is shown in the bifurcation diagram of Figure 4) until reaching a (possible) chaotic attractor, as is shown in Figure 2(d). Figures 3(a)-3(d) show the time series of $k(t)$ and $h(t)$ (the blue line and red line, respectively) for the values of $\tau_{2}$ corresponding to Figures 2(a)-2(d). 


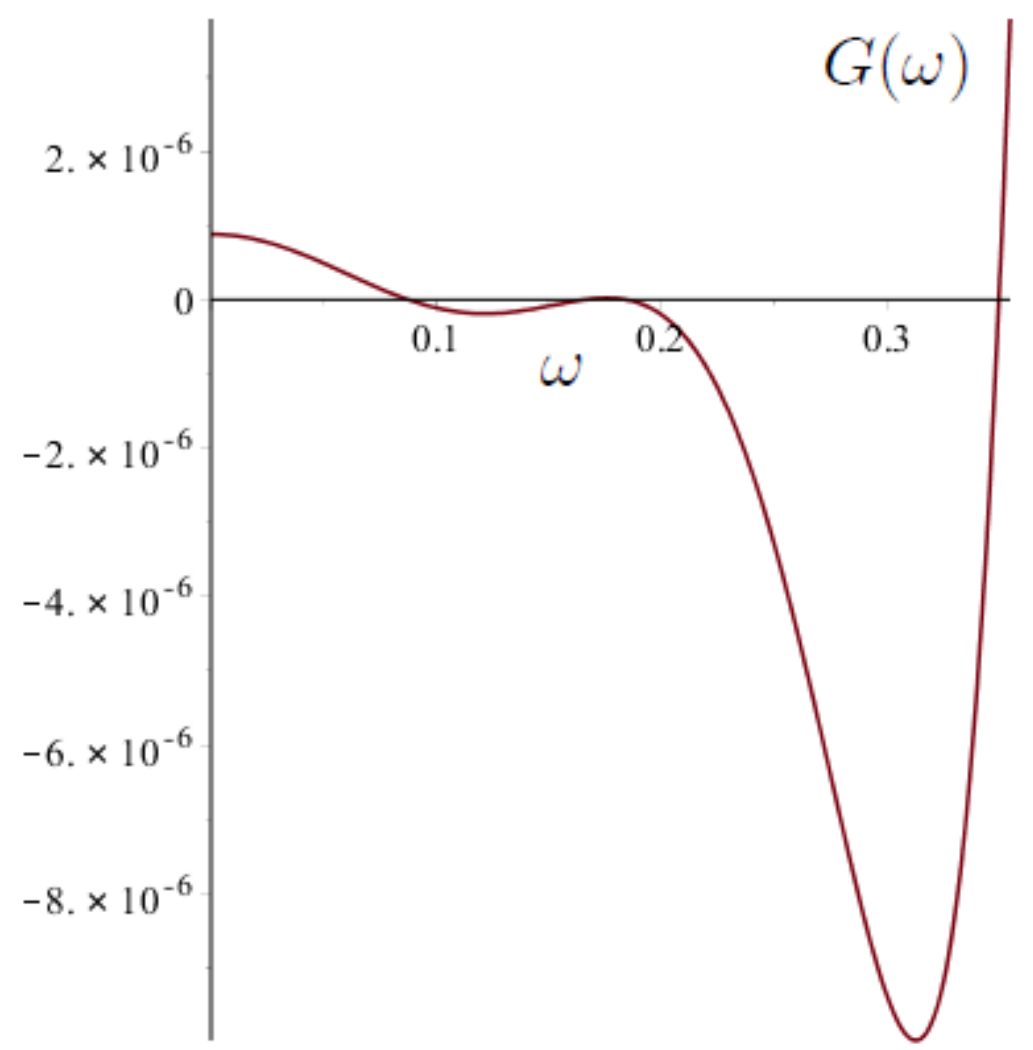

(a) 


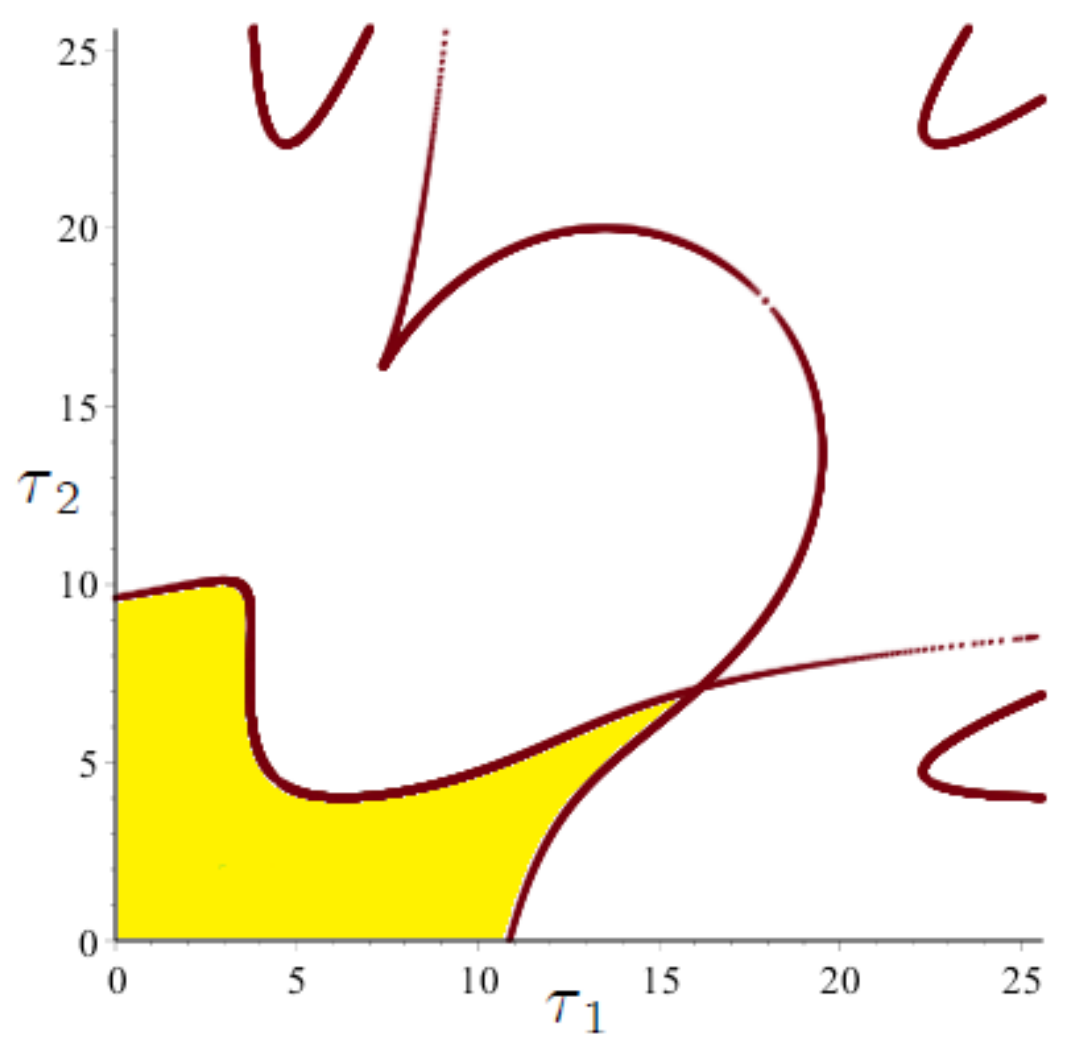

(b)

Figure 1. (a) Graph of $G(\omega)$. $G(\omega)<0$ for $\omega \in(0.0875,0.166468) \cup(0.183968,0.35)$. (b) Stability crossing curves in $\left(\tau_{1}, \tau_{2}\right)$ plane.

13 


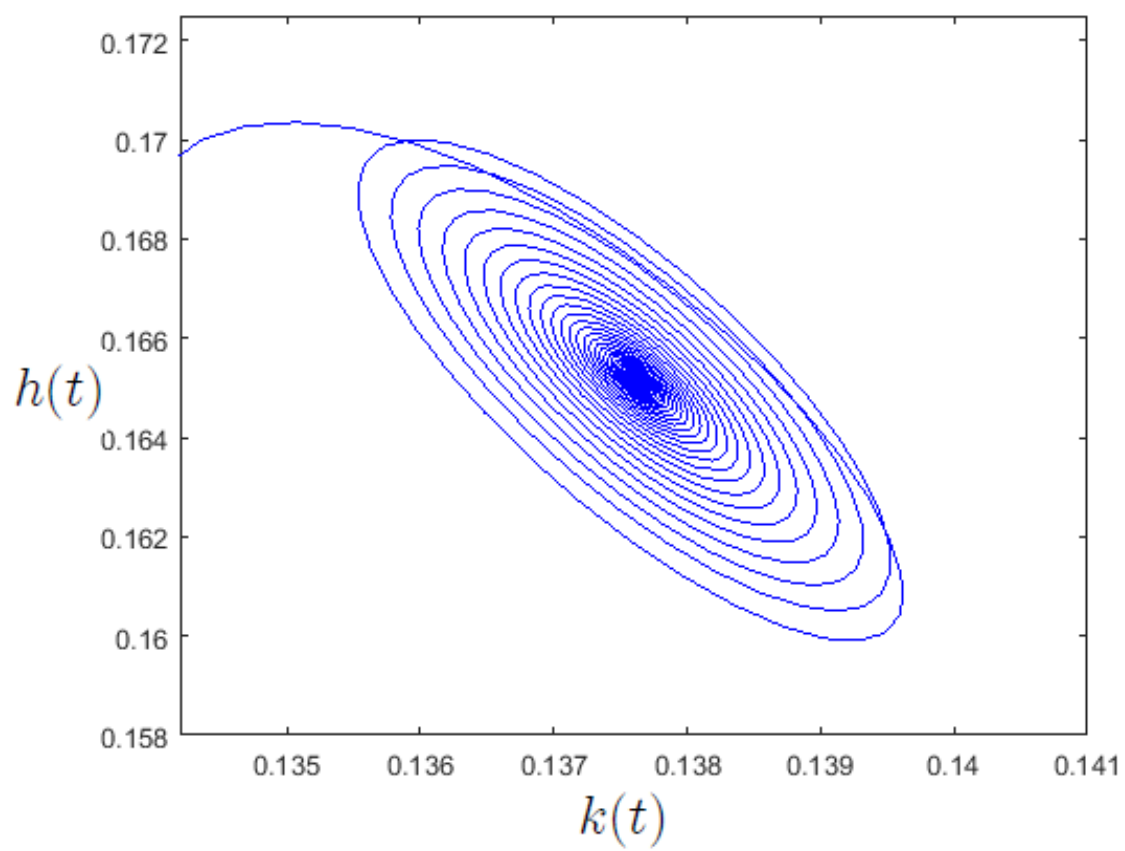

(a)

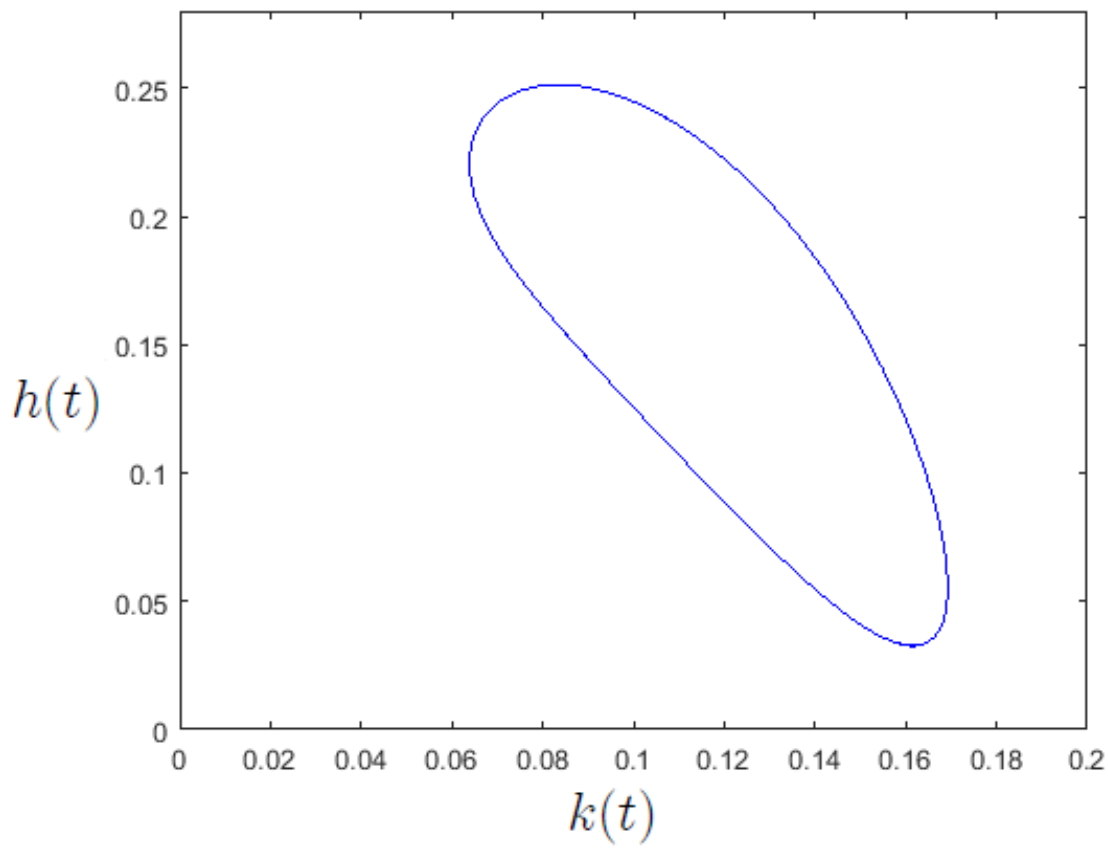

(b) 


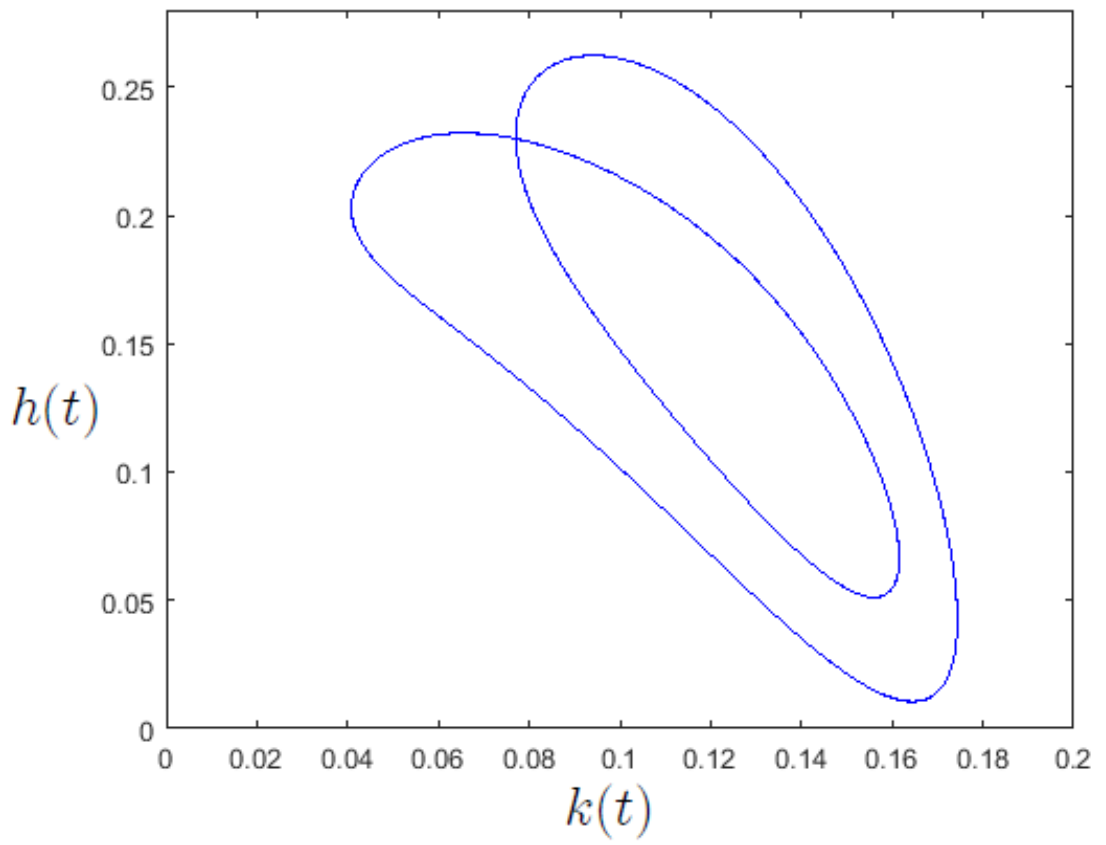

(c)

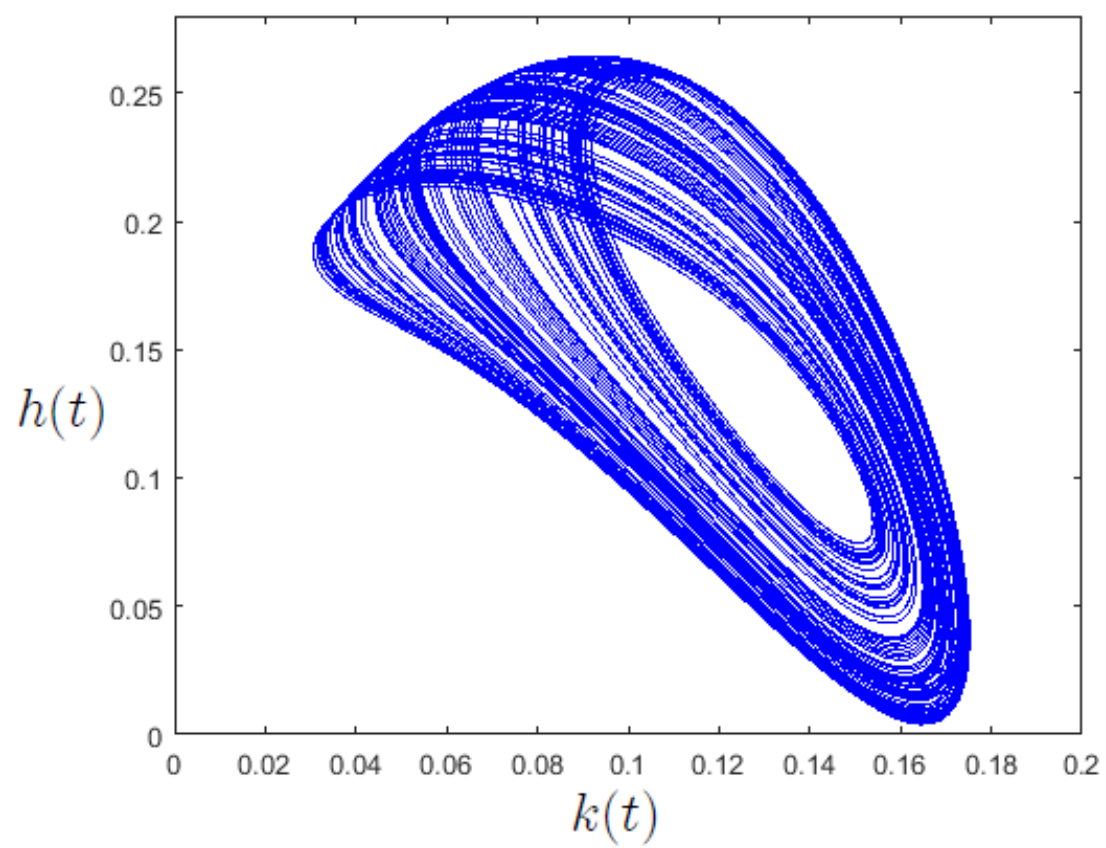

(d) 
Figure 2. Evolution of the pseudo phase plane $(k(t), h(t))$ when $\tau_{2}$ (the time delay in the production of human capital) varies. (a) $\tau_{2}=9.28$. (b) $\tau_{2}=12$. (c) $\tau_{2}=12.7$. (d) $\tau_{2}=12.89$.

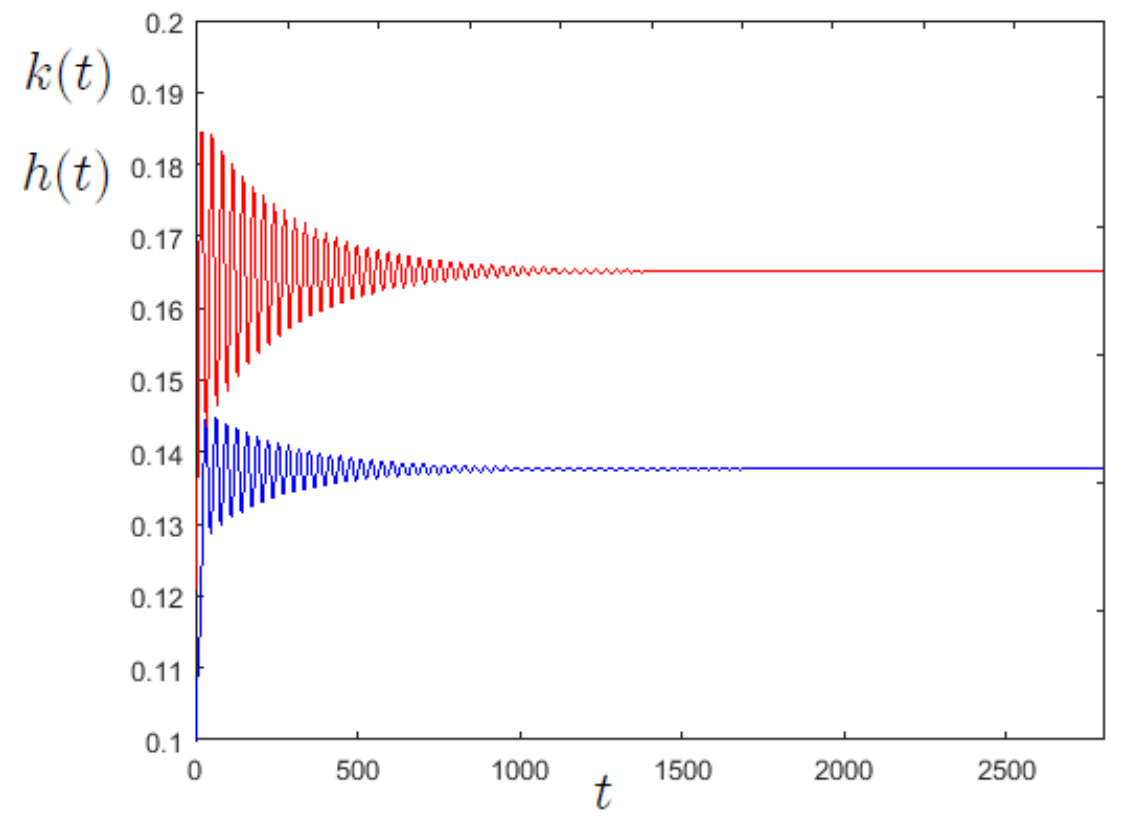

(a) 


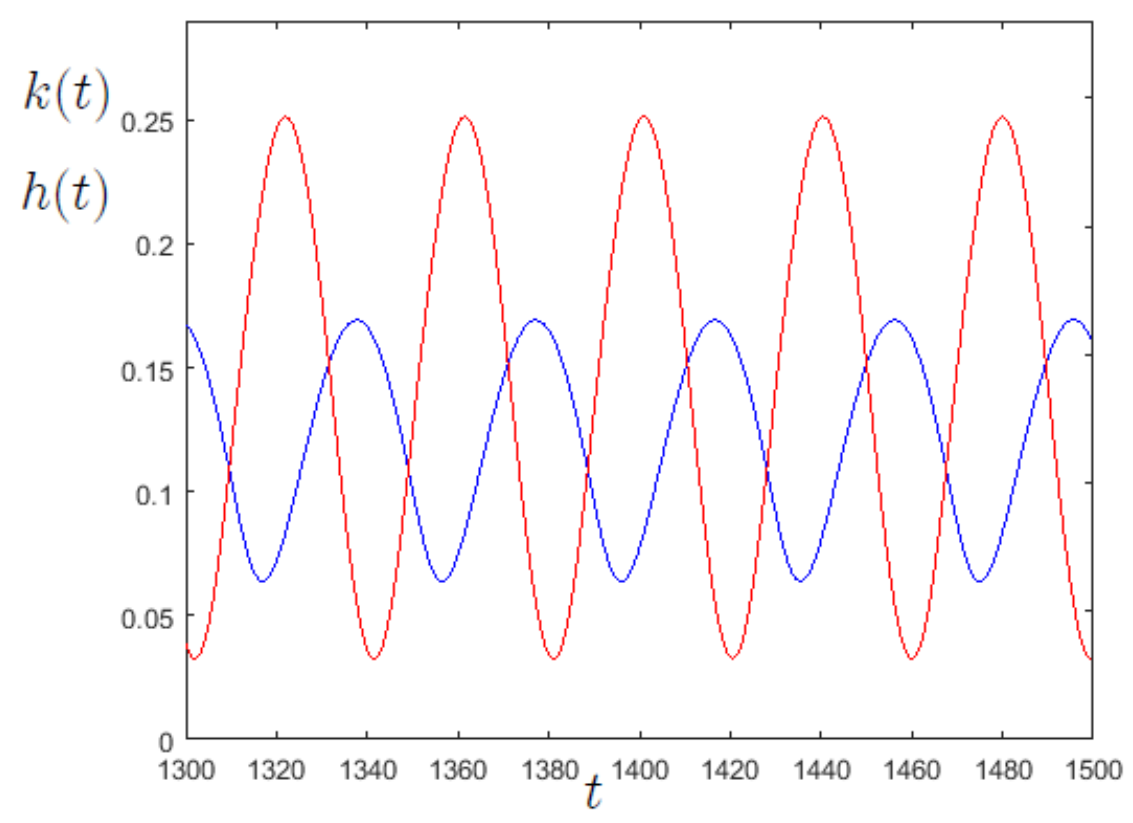

(b)

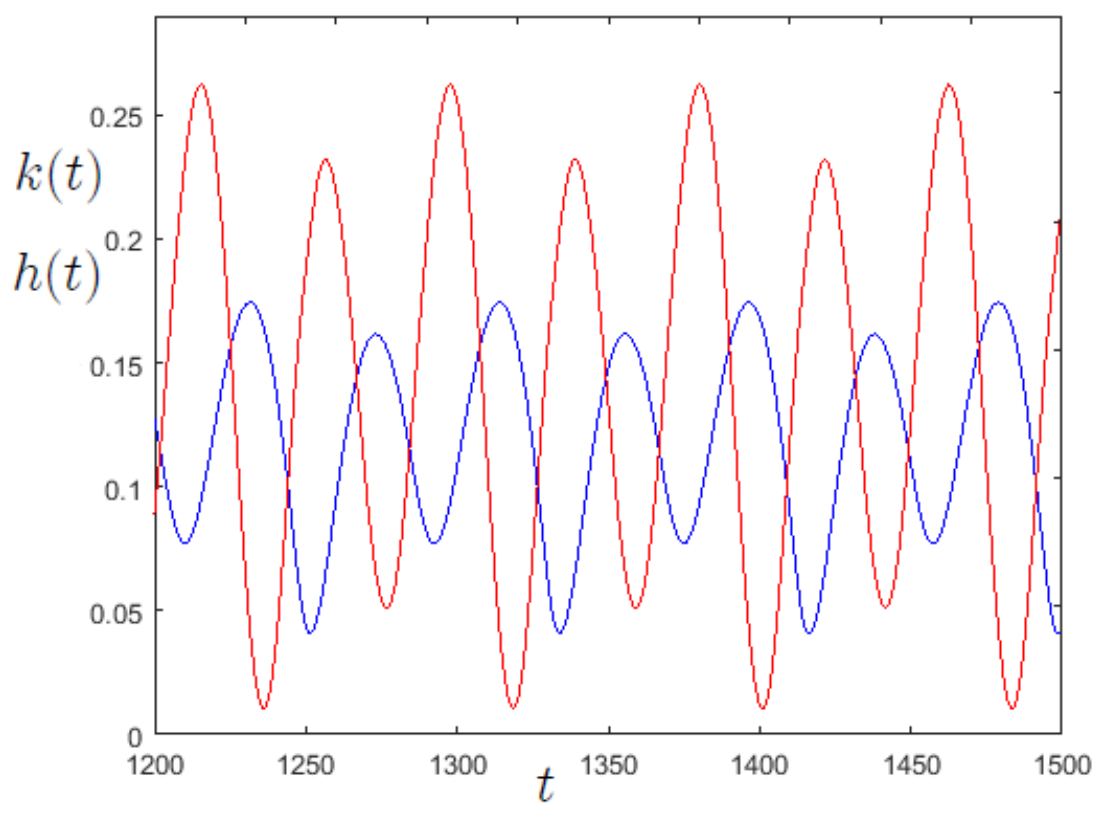

(c) 


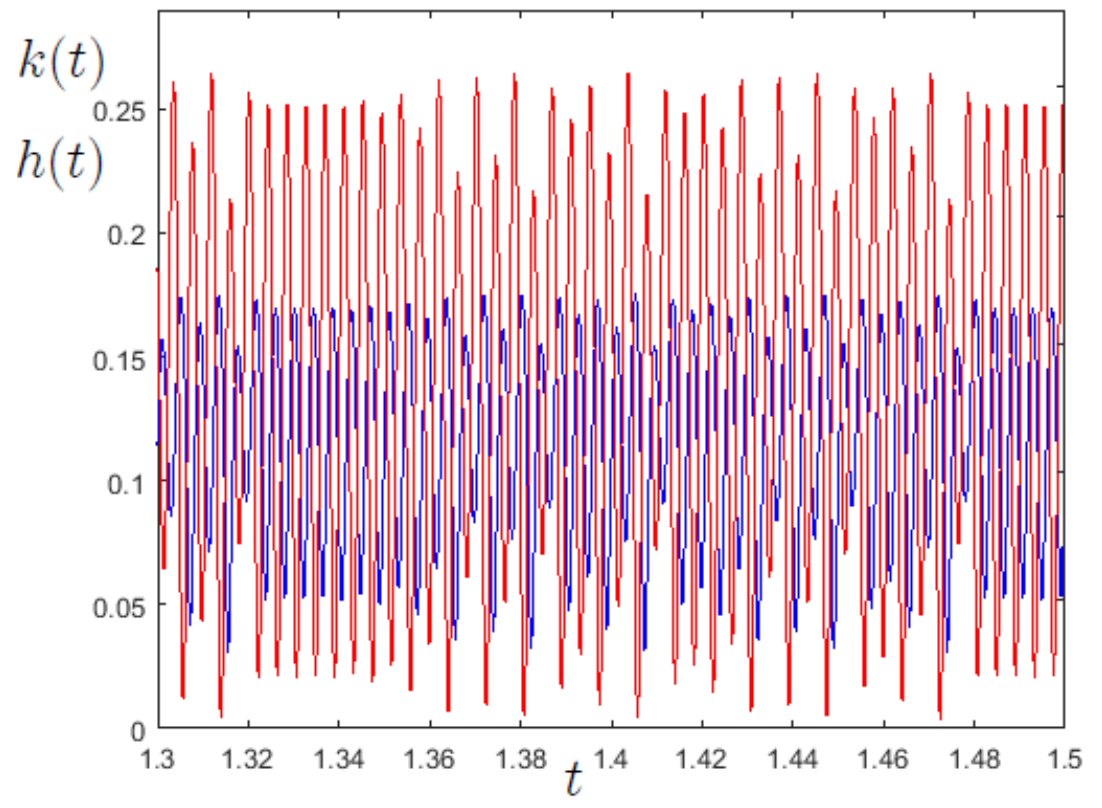

(d)

Figure 3. Time series corresponding to the values of $\tau_{2}$ in Figure 2. The blue (resp. red) line is the time series of physical (resp. human) capital. (a) $\tau_{2}=9.28$. (b) $\tau_{2}=12$. (c) $\tau_{2}=12.7$. (d) $\tau_{2}=12.89$. 


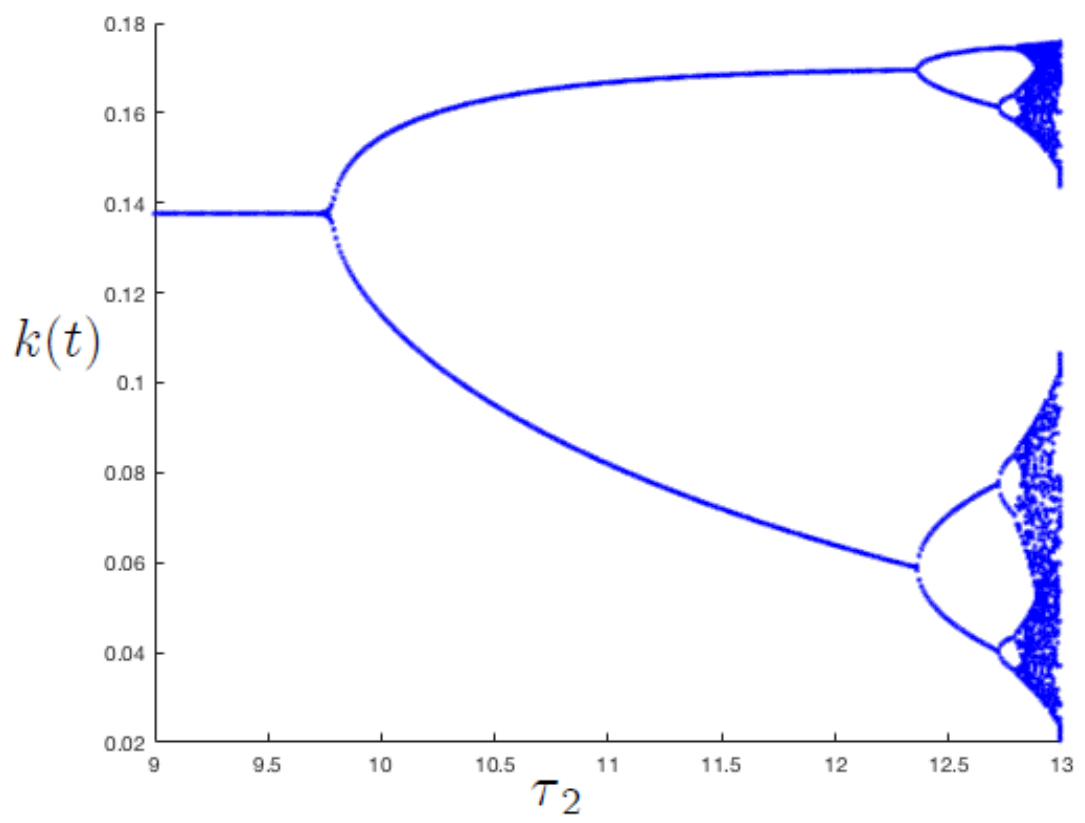

Figure 4. Bifurcation diagram for $\tau_{2}$.

Theorem 8 For small $\tau_{1}$ and $\tau_{2}$, with $\tau_{2} \in\left[0, \tau_{2,0}\right)$ satisfying $d_{0}-\left(b_{1}+c_{1}\right) b_{1}-\left(b_{1}-c_{1}\right) d_{0} \tau_{2}>0$ and $b_{1}+c_{1}-d_{0} \tau_{2}>0$, there exists $\tau_{1}^{c}>0$ such that the equilibrium $\left(k_{*}, h_{*}\right)$ of system (2) is locally asymptotically stable for $\tau_{1} \in\left[0, \tau_{1}^{c}\right)$ and unstable for $\tau_{1}>\tau_{1}^{c}$. System (2) undergoes a Hopf bifurcation at the equilibrium $\left(k_{*}, h_{*}\right)$ for $\tau_{1}=\tau_{1}^{c}$.

Proof. For small delays, $e^{-\lambda \tau_{1}} \cong 1-\lambda \tau_{1}, e^{-\lambda \tau_{2}} \cong 1-\lambda \tau_{2}$ and $e^{-\lambda\left(\tau_{1}+\tau_{2}\right)} \cong 1-\lambda\left(\tau_{1}+\tau_{2}\right)$. Then, the characteristic equation (4) reduces to

$$
\lambda^{2}+b_{1} \lambda\left(1-\lambda \tau_{1}\right)+c_{1} \lambda\left(1-\lambda \tau_{2}\right)+d_{0}\left[1-\lambda\left(\tau_{1}+\tau_{2}\right)\right]=0 .
$$

Let $\lambda=i \omega(\omega>0)$ be a root of (30). Then

$$
\omega^{2}\left(1-b_{1} \tau_{1}-c_{1} \tau_{2}\right)=d_{0}, \quad b_{1}+c_{1}=d_{0}\left(\tau_{1}+\tau_{2}\right) .
$$

Thus, we find

$$
\omega=\sqrt{\frac{d_{0}^{2}}{d_{0}-\left(b_{1}+c_{1}\right) b_{1}-\left(b_{1}-c_{1}\right) d_{0} \tau_{2}}}:=\omega_{c}, \quad \tau_{1}=\frac{b_{1}+c_{1}}{d_{0}}-\tau_{2}:=\tau_{1}^{c} .
$$

The root $\lambda=i \omega_{c}$ is a simple root of (30). Otherwise, one has

$$
2 i \omega_{c}+b_{1}\left(1-i \omega_{c} \tau_{1}^{c}\right)-b_{1} i \omega_{c} \tau_{1}^{c}+c_{1}\left(1-i \omega_{c} \tau_{2}\right)-c_{1} i \omega_{c} \tau_{2}-d_{0}\left(\tau_{1}^{c}+\tau_{2}\right)=0,
$$


and so in particular the contradiction $1-b_{1} \tau_{1}^{c}-c_{1} \tau_{2}=0$. Next, we need to verify the transversality condition. From Eq. (30), differentiating with respect to $\tau_{1}$, we get

$$
\left(\frac{d \lambda}{d \tau_{1}}\right)^{-1}=\frac{2 \lambda+b_{1}\left(1-\lambda \tau_{1}\right)+c_{1}\left(1-\lambda \tau_{2}\right)-b_{1} \lambda \tau_{1}-c_{1} \lambda \tau_{2}-d_{0}\left(\tau_{1}+\tau_{2}\right)}{\left(d_{0}+b_{1} \lambda\right) \lambda} .
$$

We conclude that

$$
\operatorname{sign}\left[\frac{d \operatorname{Re}(\lambda)}{d \tau_{1}}\right]_{\tau=\tau_{1}^{c}}=\operatorname{sign}\left[\operatorname{Re}\left(\frac{d \lambda}{d \tau_{1}}\right)^{-1}\right]_{\tau=\tau_{1}^{c}}=\operatorname{sign}\left(\frac{2 d_{0}\left(1-b_{1} \tau_{1}^{c}-c_{1} \tau_{2}\right)}{b_{1}^{2} \omega^{2}+d_{0}^{2}}\right)>0,
$$

which completes the proof.

Remark 9 Using (5) we have $d_{0}-\left(b_{1}+c_{1}\right) b_{1}=-\left[(1-\alpha)^{2}+\alpha \beta\right] \delta^{2}<0$. Hence, the condition $d_{0}-\left(b_{1}+c_{1}\right) b_{1}-\left(b_{1}-c_{1}\right) d_{0} \tau_{2}>0$ cannot hold if $b_{1}-c_{1} \geq 0$. Therefore, let us assume that $b_{1}-c_{1}<0$, i.e. $\alpha>\beta$. In this case, the conditions $d_{0}-\left(b_{1}+c_{1}\right) b_{1}-\left(b_{1}-c_{1}\right) d_{0} \tau_{2}>0$ and $b_{1}+c_{1}-d_{0} \tau_{2}>0$ are equivalent to

$$
\frac{(1-\alpha)^{2}+\alpha \beta}{(\alpha-\beta) \delta}<\tau_{2}<\frac{2-\alpha-\beta}{(1-\alpha-\beta) \delta} .
$$

In addition, notice that

$$
\frac{(1-\alpha)^{2}+\alpha \beta}{(\alpha-\beta) \delta}<\frac{2-\alpha-\beta}{(1-\alpha-\beta) \delta}
$$

if and only if

$$
(1+\alpha) \beta^{2}+\left(2 \alpha^{2}-3 \alpha-1\right) \beta+\alpha^{3}-4 \alpha^{2}+5 \alpha-1>0 .
$$

For example, this inequality is satisfied for $5-2 \sqrt{5}<\alpha<1$ since the discriminant of the corresponding equation is $\Delta=\alpha^{2}-10 \alpha+5<0$.

\section{Concluding remarks}

This article has performed a detailed study of the model of Mankiw et al. (1992) by considering time-to-build technologies. In particular, the dynamic properties of the resulting system have been studied, and the application of both the theorems on Hopf bifurcations for delayed dynamical system and recent mathematical techniques such as the stability crossing curves has allowed us to obtain several results (which are of interest also from an economic point of view). In fact, the coexistence of time delays in the accumulation of human capital and physical capital (that is, there is a time lag from the initial investment to the time in which they can be used as productive inputs) can destabilise the stationary equilibrium of the system and then make it a Solow-like model able to explain persistent fluctuations in economic variables without introducing stochastic components in the analysis (Mirman, 1973).

Further developments of the issues covered in this work can be those of endogenising individual saving behaviours by using models with optimising agents (Ramsey models) or some other approaches (e.g., kinetic models). 


\section{Acknowledgement}

The authors acknowledge two anonymous reviewers for valuable comments and suggestions on an earlier draft. The usual disclaimer applies.

\section{References}

[1] Brianzoni, S., Mammana, C., Michetti, E., 2015. Local and global dynamics in a neoclassical growth model with nonconcave production function and nonconstant population growth rate. SIAM Journal on Applied Mathematics 75, 61-74.

[2] Brock, W.A., Scott Taylor, M., 2010. The Green Solow model. Journal of Economic Growth $15,127-153$.

[3] Camacho, C., Zou, B., 2004. The spatial Solow model. Economics Bulletin 18, 1-11.

[4] Capasso, V., Engbers R., La Torre, D., 2010. On a spatial Solow model with technological diffusion and nonconcave production function. Nonlinear Analysis: Real World Applications $11,3858-3876$.

[5] Chakrabarti, B.K., Chatterjee, A., 2004. Ideal gas-like distributions in economics: effects of saving propensity. In: Application of Econophysics. Proceedings of the Second Nikkei Econophysics Symposium. Takayasu, H., Ed., Springer, Tokyo, 280-285.

[6] Chatterjee, A., Chakrabarti, B.K., Manna, S.S., 2003. Money in gas-like markets: Gibbs and Pareto laws. Physica Scripta T 106, 36.

[7] Chatterjee, A., Chakrabarti, B.K., Manna, S.S., 2004. Pareto law in a kinetic model of market with random saving propensity. Physica A 335, 155-163.

[8] Chen, H.J., Li, M.C., 2013. Child allowances, fertility, and chaotic dynamics. Chaos: An Interdisciplinary Journal of Nonlinear Science 23, 023106.

[9] Ferrara, M., Guerrini, L., Mavilia, R., 2013. Modified neoclassical growth models with delay: a critical survey and perspectives. Applied Mathematical Sciences 7, 4249-4257.

[10] Ferrara, M., Guerrini, L., Sodini, M., 2014. Stability and nonlinear dynamics in a Solow model with pollution. Nonlinear Analysis: Modelling and Control 19, 565-577.

[11] Gori, L., Sodini, M., 2014. Local and global bifurcations in an economic growth model with endogenous labour supply and multiplicative external habits. Chaos: An Interdisciplinary Journal of Nonlinear Science 24, 013122.

[12] Gu, K., Niculescu, S.I., Chen, J., 2005. On stability crossing curves for general systems with two delays. Journal of Mathematical Analysis and Applications 311, 231-253.

[13] Guerrini, L., 2006. The Solow-Swan model with a bounded population growth rate. Journal of Mathematical Economics 42, 14-21.

[14] Hale, J., 1977. Theory of Functional Differential Equation. Springer, New York. 
[15] Lin, X., Wang, H., 2012. Stability analysis of delay differential equations with two discrete delays. Canadian Applied Mathematics Quarterly 20, 519-533.

[16] Mankiw, N.G., Romer, D., Weil, D.N., 1992. A contribution to the empirics of economic growth. Quarterly Journal of Economics 107, 407-437.

[17] Merton, R.C., 1975. An asymptotic theory of growth under uncertainty. Review of Economic Studies 42, 375-393.

[18] Mirman, L.J., 1973. The steady state behavior of a class of one sector growth models with uncertain technology. Journal of Economic Theory 6, 219-242.

[19] Naimzada, A., Pireddu, M., 2014. Dynamics in a nonlinear Keynesian good market model. Chaos: An Interdisciplinary Journal of Nonlinear Science 24, 013142

[20] Prandini, J.C., 1994. The limiting behaviour of Solow's model with uncertainty when the variance goes to zero. Economic Theory 4, 799-809.

[21] Richmond, P., Mimkes, J., Hutzler, S., 2013. Econophysics \& Physical Economics. Oxford University Press, Oxford.

[22] Ruan, S.G., Wei, J.J., 2003. On the zero of some transcendental functions with applications to stability of delay differential equations with two delays. Dynamics of Continuous, Discrete and Impulsive Systems Series A: Mathematical Analysis 10, 863-874.

[23] Schenk-Hoppé, K.R., Schmalfuß, B., 2001. Random fixed points in a stochastic Solow growth model. Journal of Mathematical Economics 36, 19-30.

[24] Solow, R.M., 1956. A contribution to the theory of economic growth. Quarterly Journal of Economics 70, 65-94.

[25] Zak, P.J., 1999. Kaleckian lags in general equilibrium. Review of Political Economy 11, 321330. 